\title{
L'auteur réincarné ou de l'interprétation comme folie. À propos de La Guérison de Roberto Gac
}

Sophie Rabau

\section{OpenEdition}

\section{Journals}

Édition électronique

URL : http://journals.openedition.org/recherchestravaux/1300

DOI : 10.4000/recherchestravaux.1300

ISSN : 1969-6434

Éditeur

UGA Éditions/Université Grenoble Alpes

\section{Édition imprimée}

Date de publication : 15 mai 2004

Pagination : 173-185

ISBN : 2-9518254-3-9

ISSN : 0151-1874

Référence électronique

Sophie Rabau, «L'auteur réincarné ou de l'interprétation comme folie. À propos de La Guérison de Roberto Gac », Recherches \& Travaux [En ligne], 64 | 2004, mis en ligne le 20 mai 2019, consulté le 08 septembre 2020. URL : http://journals.openedition.org/recherchestravaux/1300 ; DOI : https://doi.org/ 10.4000/recherchestravaux.1300 


\section{L'auteur réincarné ou de l'interprétation comme folie À propos de La Guérison de Roberto Gac}

S'il est un paradoxe dans La Guérison de Roberto Gac, il est du lecteur plus que de l'auteur. Car ce livre en deux volumes est illisible dans sa globalité et offre des parcours de lecture qui varient en fonction des compétences linguistiques de chaque lecteur. Le premier volume est en effet écrit en quatre langues - français, italien, anglais et espagnol, sans oublier quelques mots d'araucan, épars ici et là. Le deuxième volume se présente comme la "Traduction en français des textes de La Guérison écrits en italien, en anglais et en castillan". Selon que le lecteur maîtrise plus ou moins bien ces différentes langues, il lira donc la même histoire mais dans des idiomes différents et, à moins d'être pris d'un scrupuleux souci de vérification de toutes les traductions en français, il ne lira jamais la totalité de l'ouvrage, tout en le comprenant pourtant parfaitement.

Mais si ce livre est paradoxalement écrit pour ne jamais être lu en son entier par un seul lecteur, c'est le fait d'un auteur, non pas tant de Roberto Gac que de son héros, et néanmoins auteur s'il en fut. Il s'agit de Dante Alighieri dont La Guérison narre la réincarnation au XXe siècle sous les traits d'un médecin chilien métissé de sang indien, comme nous l'apprenons dès la première phrase du premier volume:

Quitte à t'agacer ou à te décevoir, il vaut mieux lecteur - que je te dévoile tout de suite mon secret: je ne suis pas du tout celui que l'on croit. Non. Je suis bel et bien Dante Alighieri réincarné et cette histoire que tu commences à lire est une nouvelle Divine Comédie ${ }^{\text {I. }}$ (p.9)

I. Sauf indication contraire, nous nous référons au premier volume de Gac, R., La Guérison, Paris, éditions de la Différence, 2000. 
Chilien d'origine indienne, Dante réincarné parle naturellement l'espagnol et l'araucan; dès qu'il apprend qu'il fut, dans une autre vie, un grand poète toscan, il lui faut - ne serait-ce que pour prendre connaissance de son œuvre passée, "réapprendre" l'italien, ce qu'il fait avec une "troublante facilitée (p. I5). On ne sait pas d'où le " toubib" (p.9) chilien tient sa connaissance du français et de l'anglais, mais ce sont en tout cas les théories construites dans sa vie et son œuvre antérieures qui justifient ce mélange linguistique: se reportant au traité De Vulgari Eloquentia, il rappelle en effet que pour lui la langue d'oïl (soit le français) est adaptée à la prose tandis que les langues du sí (le toscan et le castillan) sont plus " appropriées à la poésie ${ }^{2}$ " (p. 19). Enfin l'anglais "langue du iö (ja ou yes)" sera également utilisée car cette langue "hypocritement romanesque" est "excellente pour englober et occulter tous types d'obscénités» (p. 19): c'est ainsi que certains passages de La Divine Comédie seront cités en anglais et non en italien.

Ainsi averti qu'il ne pourra pas tout lire et qu'il lira en allant d'une langue à l'autre, voire d'un volume à l'autre, le lecteur de Gac peut toutefois espérer lire plus facilement et mieux comprendre, sinon La Guérison, du moins une autre œuvre, tout aussi complexe: celle qui fut écrite au XIII' siècle par un certain Dante Alighieri. Car, par le miracle de la fiction, se voit réalisé ce qui pourrait bien être le vœu secret de tout interprète: un accès direct et immédiat à l'intériorité de l'auteur qui laisserait transparaître son intention et par là livrerait, sans que nous n'ayons plus à le chercher entre les lignes et les symboles, le sens qu'il voulut donner à son œuvre. Alors l'intention ne serait plus cette hypothèse dont la reconstitution est toujours hasardeuse, voire illusoire, mais l'expression d'un véritable projet qui rendrait compte de l'œuvre, permettrait même de mesurer l'écart entre le résultat et le dessein de l'auteur: gageons que même le zélateur le plus fervent de la clôture textuelle, même le défenseur le plus ardent de la déconstruction ou de l'intentio lectoris (Eco 1993) ne dédaignerait pas quelques révélations livrées par l'auteur lui-même, ne serait-ce que pour renforcer par l'autorité du créateur ce que la seule étude des formes avait déjà livré. Que dire de lectures plus traditionnellement philologiques dont le dessein avoué n'est autre que de reconstituer l'intention de l'auteur: tout discours de l'auteur sur son œuvre est un éclaircissement, toute

2. Voir aussi cette justification plus métatextuelle du mélange linguistique (p.20): «le français joue le rôle de la langue populaire; l'espagnol, celui de la langue de la folie (Don Quichotte) et, simultanément, le rôle de la psychothérapie (Sancho Panza); l'anglais, le rôle de langue vénale des "businessmen "; l'araucan et le latin, le rôle des acteurs comiques; finalement, l'italien joue le rôle de la langue la plus noble et poétique de toutes, la langue maternellew. 
observation de l'intimité de l'auteur au travail fait en soi explication: le travail herméneutique est alors une "reconstitution de l'activité intérieure de l'auteur quand il compose", selon la formule de Schleiermacher (I829). Or cette reconstitution n'est qu'un ersatz qui n'aurait plus de raison d'exister si l'auteur était ici face à moi et me parlait lui-même de son œuvre.

Pour être fictionnel, le récit de Gac ne se lit donc pas moins comme une réponse à ce désir, ou plutôt ce fantasme, herméneutique, d'une interprétation non plus par l'autre mais par le même, par l'auteur lui-même. Et la fiction serait le lieu où s'expérimenterait le bien-fondé de cette opinion selon laquelle l'auteur est l'exclusif dépositaire de l'ultime vérité de son œuvre. C'est en ce sens que la représentation de l'auteur y est paradoxale au sens étymologique où elle déçoit l'opinion commune. Certes, Dante réincarné interprète effectivement son œuvre et se trouve effectivement représenté comme le dépositaire de clefs qu'il est le seul à connaître. Mais il n'en est pas moins également une incarnation, voire une allégorie, de l'impuissance herméneutique. L'auteur, s'il revenait, ne pourrait pas, lui non plus, dire le sens de son œuvre: c'est de ce paradoxe que la fiction de Gac offre l'argument et la représentation expérimentale.

\section{De l'autobiographie fictive à la fiction d'un discours auctorial}

Pour dessiner les contours de ce paradoxe, il convient de lire La Guérison non seulement comme une autobiographie fictive, ce qu'elle est à un premier niveau, mais également comme une représentation fictionnelle de l'auteur, comme la fiction d'un discours sur et de l'auteur.

A première lecture, La Guérison est une autobiographie fictive, à michemin entre le récit d'initiation ou de formation et la tradition picaresque. Deux fils autobiographiques s'entrecroisent: Dante réincarné raconte en alternance sa vie au XX' siècle et son existence au Moyen Âge. Dans les deux cas, on lit en parallèle l'histoire d'une initiation et d'un apprentissage, puisque les aventures du Dante contemporain sont la répétition «modernisée » de la vie du premier Dante. Ainsi les deux Dante doivent-ils apprendre à devenir Dante, chacun dans un contexte différent.

Afin de devenir le grand poète italien que l'on sait, Dante première version doit surmonter "la ténébreuse dépression" (p. I7) que provoque en août I290 la mort de Beatrice, et s'initier aux arcanes de la politique florentine, sans négliger l'art et la science de son temps. Dante réincarné vit une expérience similaire: après le départ pour les Etats-Unis, ironiquement appelés American Paradise (p.17), de sa maîtresse américaine, une certaine 
Beatrice, il tombe à son tour dans un désespoir qui le pousse à essayer un "philtre végétal ». Il s'ensuit un coma dont il se réveille fort d'une seule certitude: il est la réincarnation de Dante Alighieri, dont il a lu La Divine Comédie sur le conseil de Beatrice. Il doit donc écrire "une nouvelle comédie» (p. I8) qui lui permettra de retrouver les faveurs de Beatrice et aussi de lutter contre une secte, la Société des Hommes Célestes, dont elle est un membre actif. Bien sûr, cette décision est interprétée par ses médecins comme un symptôme psychotique des plus inquiétants et nous pouvons donc lire la suite des aventures de Dante 2 comme l'histoire d'un fou qui se prend pour Dante, ou comme l'histoire de Dante réincarné que l'on prend pour un fou. Mais dans les deux cas, c'est un apprentissage de l'état de poète, ou de fou se prenant pour un poète, qui nous est donné à lire. Or, dans l'hôpital psychiatrique où il est interné, Dante 2 est guidé dans cet apprentissage par un médecin dont le nom semble plaider pour la thèse de l'authentique réincarnation, puisqu'il se nomme le Docteur Virgilio Pazzi et se trouve être d'origine italienne (p. I8): le texte que nous lisons est donc bien une nouvelle Comédie, traversée de l'enfer de la folie sous la direction de Virgilio. Et la "guérison" psychiatrique est l'équivalent contemporain de la rédemption.

Cet apprentissage est loin d'être éthéré et les deux Dante de Gac partagent aussi un goût prononcé pour le sexe (voire, dans le cas de Dante I, pour le sexe incestueux), l'alcool, la bonne chère et les terrestres avantages que procure la gloire. Le récit de formation est donc aussi un récit circonstancié des plaisirs terrestres dont jouissent sans aucun scrupule Dante 2 mais surtout Dante I qui n'hésite pas à faire jouer intrigues rouées et ruses hypocrites pour parvenir à ses fins. Autobiographie fictive dont le narrateur fait l'inventaire des plaisirs charnels et des ruses, $L a$ Guérison tient donc aussi du picaresque. La thématique dantesque de la rédemption prend alors une dimension moins chrétienne qu'il ne semble: comme dans le Lazarillo de Tormes ou dans Moll Flanders, la perspective d'une rédemption finale semble plus un prétexte pour raconter, non sans complaisance, ses nombreux péchés qu'une sincère préoccupation.

Mais cette double autobiographie fictive ne peut être réduite ni au roman de formation, ni à la tradition picaresque, car le personnage qui y raconte sa vie est aussi un auteur. Dès la première phrase du roman, la réincarnation est étroitement liée à l'entreprise d'écriture: être Dante réincarné, c'est écrire à nouveau La Divine Comédie et c'est évoquer une vie médiévale où l'on fut d'abord un auteur. Surtout, La Guérison s'inscrit clairement dans une catégorie de textes que nous appellerons "fiction d'auteur" et qui se caractérise par les traits suivants: le texte est contractuellement fictionnel, mais repré- 
sente un auteur historiquement attesté dans les histoires de la littérature; l'auteur semble représenté afin de combler un double manque que peut causer l'expérience de lecture, manque de la présence de l'auteur au sens où l'auteur n'accompagne jamais physiquement son texte, manque herméneutique au sens où l'auteur n'est pas là pour m'aider à comprendre son texte au moment où je le lis; enfin, la fiction d'auteur prend une forme généralement hypertextuelle: le portrait de l'auteur découle de son œuvre comprise comme le reflet de la vie que l'on invente pour son auteur ${ }^{3}$.

La Guérison répond parfaitement à ces trois critères et appartient donc à la catégorie de la fiction d'auteur, comme, entre autres exemples contemporains, Las Vidas escritas de J. Marías, Rimbaud le Fils de Michon, ou Le Roman de Monsieur Molière de Boulgakov. En effet, l'autobiographie fictive est indissociable de la fiction, d'abord parce qu'elle autorise une invention de l'auteur historique, ensuite parce que le genre même de l'autobiographie fictive découle de l'œuvre de Dante, enfin parce qu'elle semble réparer l'absence de l'auteur qui révèle et son intimité et le sens de son œuvre. Le texte est contractuellement fictionnel, ce que suggère l'indication "roman " portée sur la couverture et ce que confirme l'invraisemblance même de la réincarnation; or, c'est dans le cadre de cette fiction qu'il est question de Dante Alighieri dont l'existence historique est attestée. Ensuite, la représentation autobiographique de Dante découle ici évidemment de son œuvre plus que de toute autre source historique. La fiction de Dante écrite par R. Gac.est donc dans un rapport hypertextextuel et intertextuel à l'œuvre de Dante: sur un plan générique, le choix même d'écrire une autobiographie fictive fait référence à La Divine Comédie mais aussi à la Vita Nuova. Sur un plan structurel, l'œuvre de Dante fonde l'organisation de La Guérison: les aventures de Dante réincarné suivent la composition tripartite de La Divine Comédie. La première partie où Dante raconte au docteur Virgilio son épisode de folie, correspond à L'Enfer, comme l'indique sans équivoque la phrase de Virgilio qui clôt cette partie "ya hemos vivido la parte más oscura, más infernal de su enfermedad" (p. I52). La deuxième partie, où le docteur Virgilio reste présent mais se fait plus discret dans ses interventions, correspond au Purgatoire, puisqu'aussi bien elle se clôt par le départ de Virgilio, qui, après avoir suggéré à son patient de se faire passer pour romancier afin qu'on ne le prenne pas pour un fou, lui annonce qu'il ne pourra plus l'accompagner par la suite (p. 260). La dernière partie peut se lire comme

3. Nous renvoyons ici à une recherche en cours sur cette notion. Pour une première approche nous nous permettons de renvoyer à Rabau (200I) et, pour une approche similaire, à Puech (2000). 
l'équivalent du Paradis au sens où Dante $2 \mathrm{y}$ raconte trois aspects édéniques de sa vie médiévale: l'accueil en effet paradisiaque qu'il reçut dans "le superbe château" de son "nouveau protecteur", la rédaction du Paradis et enfin sa mort et son arrivée au vrai paradis où l'on apprend l'origine du livre que nous sommes en train d'achever: saint Paul n'admet Dante que s'il s'engage à lui consacrer une meilleure place dans la nouvelle Comédie qu'il écrira à l'occasion d'une réincarnation dans une vie future. Cette organisation est soulignée par l'évolution du style, de plus en plus limpide, du récit. Cet éclaircissement progressif reproduit l'impression de lecture que peut donner la Nouvelle Comédie, comme cela est d'ailleurs suggéré: «la lecture deviendra progressivement - suivant les rythmes eschatologiques de $L^{\prime} E n f e r$, du Purgatoire et du Paradis - moins saccadée, plus claire et plus rapide ${ }^{4}$ (p. 2o). Enfin, l'œuvre de Dante structure également le livre de Gac parce que La Guérison se donne comme un long récit entrecoupé de citations de Dante: ces citations ont parfois fonction d'illustration en ce qu'elles expriment la situation où se trouve le héros-poète et elles sont parfois objet d'une explication quand Dante 2 révèle ce qu'il voulut dire ou dans quelles circonstances il écrivit ces lignes. Ce dispositif est emprunté à la Vita Nuova qui se présente précisément comme un récit autobiographique en prose écrit par Dante pour recontextualiser des œuvres poétiques écrites pour l'amour de Beatrice 5 . Le Dante qui nous est donné à voir est donc le fils autant que le père de son œuvre et, selon la formule de Puech (2000:7), sa vie devient " un des tomes de son œuvre complète » tant le récit de Gac pourrait être écrit par Dante. Le choix de l'autobiographie renforce évidemment cette impression: non seulement on retrouve l'œuvre de Dante, mais en outre, dans la fiction, on lit en effet une œuvre de Dante.

Enfin l'autobiographie fictive sert la fiction d'auteur au sens où elle offre une remarquable réparation de l'absence de l'auteur. En effet, le choix même de l'autobiographie semble avoir pour fonction de combler fort efficacement une expérience du manque: l'auteur prend lui-même la parole pour révéler son intimité et ses secrets, et, de surcroît, pour élucider le sens de son œuvre. En outre, l'idée même de la réincarnation semble avoir pour seule fonction

4. Cet éclaircissement progressif vient surtout de ce que la première partie se présente comme un enchevêtrement entre le récit à Virgilio, écrit en espagnol, italien et anglais du "délire " de Dante 2, et le début du récit de sa vie médiévale écrite en français. Or, plus on progresse vers la troisième partie et plus les dialogues entre Dante 2 et Virgile se font rares, pour finalement disparaître: le texte évolue donc vers un récit linéaire de plus en plus limpide, tout entier consacré à la vie médiévale de Dante.

5. Cette parenté avec la Vita Nuova est d'ailleurs revendiquée p. I7r. 
de redonner une présence et un présent à un auteur absent dont il ne reste que les pages écrites. C'est aujourd'hui, ici, et maintenant que Dante fait ces révélations. La métaphore de l'auteur "vivant", "de retour parmi nous", "présent» ou encore "ressuscité ${ }^{6}$ " est ici littéralisée: Dante est vraiment ressuscité, prêt à nous livrer les détails les plus intimes de sa vie et de ses choix poétiques. Le caractère picaresque de l'autobiographie prend alors un autre sens: l'auteur est non seulement présent, mais de surcroît prêt à dévoiler ses plus bas instincts, ses désirs charnels les plus secrets: le discours sur le plaisir est aussi une manière de donner à voir l'intimité de l'auteur, de révéler ce qui, dans l'œuvre, n'est pas dit. Dans La Guérison, l'auteur parle de soi et de son œuvre. Et l'on s'attend donc à mieux comprendre et l'homme et l'œuvre ou, plutôt, à mieux comprendre l'œuvre grâce à l'homme.

\section{Dante interprète de Dante: un éclaircissement}

Dante parlant de Dante est le meilleur interprète possible de son œuvre au XX' siècle. Voilà la morale apparente de La Guérison, ce que sous-tend une poétique de la révélation: seul Dante peut dire ce que nous devrions mais ne pouvons savoir pour comprendre son œuvre en fonction de l'intention de l'auteur.

La poétique de la révélation passe d'abord par un décalage efficace entre l'image traditionnelle de Dante et le récit que lui prête Gac. Ainsi la trivialité des thèmes, entre sexualité, nourriture et autres plaisirs charnels contraste avec l'idée plus éthérée d'un Dante chantre de la chasteté et poète de la chrétienté. En outre, l'effet de révélation passe par ce que l'on pourrait appeler une personnalisation de l'étiologie: là où nous aurions tendance à expliquer certains passages de l'œuvre par des catégories morales ou philosophiques générales, le Dante de Gac donne des explications beaucoup plus personnelles qu'il est évidemment le seul à pouvoir livrer. Ainsi on apprend que tels vers du purgatoire qui font l'éloge de la chasteté furent écrits sous le coup d'une déception aussi sexuelle qu'incestueuse: Antonietta fille de Dante, vient de se refuser à son poète de père (p. 275). Enfin, l'effet de révélation vient aussi de la personnalité prêtée à la réincarnation de Dante: métis indien, hispano-américain, et plus paillard que pieux, ce nouveau Dante s'oppose terme à terme à l'image d'un Dante poète de la civilisation, de la

6. On trouvera sur la page de couverture de Puech (2000) un beau florilège d'exemples de ces métaphores dans le discours sur l'auteur. 
tradition occidentale et de la chrétienté. En somme, si Dante revenait, nous ne le reconnaitrions pas, et lui seul peut se reconnaître comme Dante. On pense ici à l'Homère troglodyte et analphabète que décrit Borges dans "l'Immortel ». Homère ne ressemble pas plus à Homère que Dante ne ressemble à Dante. Cette distance affole nos certitudes d'interprète. Si Dante réincarné est tellement éloigné de l'image que nous avons de lui, sommesnous vraiment sûrs de connaître Dante et de comprendre son œuvre? N'estil pas aussi éloigné de nous que le serait un chilien paillard métissé de sang indien? Notre lecture de Dante n'est-elle pas une terrible méprise où nous prendrions l'autre pour le même? Mais dans ce cas seul l'auteur est à même de corriger la méprise, de s'imposer comme autre là où nous attendons le même.

Cette écriture de la révélation touche surtout l'élucidation de l'œuvre. Être Dante permet de révéler ce qu'a voulu dire Dante: Dante 2 sait ce qu'a voulu faire Dante I. Les choix poétiques de La Divine Comédie sont ainsi expliqués de l'intérieur, comme au début du chapitre IV (p.49) où le poète rappelle qu'il "savait bien" qu'il aurait "dû élaborer trois formes poétiques distinctes en accord avec la spécificité de chacune de ces trois contrées", puis justifie ses choix passés (p. 5o) tout en mettant en lumière la structure de son œuvre médiévale et par là de son œuvre contemporaine, nouvelle Divine Comédie organisée sur le modèle de l'ancienne. De manière plus anecdotique, un sonnet en apparence tout à fait éthéré se voit donner un sens franchement sexuel, tandis que l'identité de la Donna Pietosa, figure mystérieuse de la Vita Nuova, est enfin dévoilée. La réincarnation a donc une fonction herméneutique clairement revendiquée:

Durante siglos, los dantólogos se han pregutntado quién es la Donna Pietosa, la muchacha que con su mirada de amor me ayudó a salir de la locura en el Medioevo. Paralelamente, ninguno sabe quién es Lisetta. Pues bien, he tenido que reencarnarme para, entre otras cosas, dejar bien en claro ese tipo de detalles: Lisetta y la Donna Pietosa son una sola y misma personna... (p. 172).

L'explication de l'œuvre par son auteur réincarné est particulièrement efficace pour trois ensembles de raisons. Premièrement, même si Dante 2 répète seulement ce que l'on sait déjà, les informations qu'il donne passent du statut d'hypothèse interprétative à celle de faits établis: l'auteur a vraiment voulu cet effet, puisqu'il s'en souvient et le dit. Ensuite, la situation particulière de Dante fait de lui une sorte d'interprète idéal, au sens où il appartient pleinement à son époque et pleinement aussi au Moyen Âge dont il possède une mémoire absolue. Ainsi, il est capable de convoquer les théories du "texte moderne" pour expliquer ses choix poétiques médiévaux (p. 5o). Or l'identité entre l'interprète et l'auteur semble abolir tout danger 
d'anachronisme: au lieu que l'interprète impose son siècle à l'auteur, discours auctorial et discours herméneutique fusionnent, ne formant plus qu'un seul propos qui appartient indifféremment au passé et au présent. Enfin, l'auteur réincarné possède un pouvoir de dévoilement: il est présenté comme le dépositaire d'un secret que lui seul peut révéler et son interprétation est donc par définition supérieure, quand bien même elle est incongrue. En somme, les limites et écueils habituels de l'interprétation - statut conjecturel, anachronisme, erreur d'appréciation, sont abolis par la seule identité auctoriale de l'interprète. L'auteur-interprète est donc par définition supérieur à tout autre spécialiste de Dante; Dante est le meilleur des Dantologues. Bien plus, en un ultime paradoxe, Dante est le seul capable de résister à l'autorité de Dante, en l'espèce de ne pas accorder foi à ses écrits, de livrer sur sa vie des informations contraires à ce qu'il a écrit (p.165). En somme, le roman de Gac semble répondre à l'attente d'un lecteur exigeant, avide de corrections et de divulgations opposées à la vulgate qui court sur Dante, comme cela est finalement dit à l'avant-dernière page du livre:

Peut-être, lecteur, voudrais-tu que je dénonce maintenant, un par un, les écrivailleurs, critiquailleurs et littérateurs en tout genre ( $y$ compris les politiciens et les curés) qui par jalousie, envie, petitesse morale, ignorance, mesquinerie intellectuelle ou bêtise, ont répandu ou continuent à répandre des clichés, des lieux communs et des niaiseries sur moi et sur mon œuvre en toute impunité. (p. 3II)

Les auteurs qui inventent la vie d'auteurs historiquement attestés aiment à se présenter comme les dépositaires d'une vérité qu'ils sont les seuls à connaitre. Dans Sogni di sogni, Tabucchi narre les rêves d'autres auteurs; dans Les Vies imaginaires, Schwob livre la vraie version de la mort de Pétrone tandis que dans Vidas Escritas, Marías annonce que Faulkner n'a pu mourir d'une thrombose mais assurément d'une chute de cheval. En donnant la parole à Dante, Roberto Gac a trouvé le moyen le plus sûr et le plus absolu de mettre en œuvre cette écriture de la révélation: ce qu'il invente est vrai, plus vrai que tout ce qui jamais a été dit, puisque c'est Dante qui le dit.

Un fait gênant vient toutefois troubler ce beau dispositif. Le roman de Gac est une fiction, ni plus ni moins que les autres textes que nous venons de citer. Et ce roman s'attaque autant à des discours critiques qu'à d'autres fictions, mettant sur le même plan discours référentiels et fictionnels: quand Dante 2 évoque les «dantologues» dont il conviendrait de corriger les affirmations, le nom de Balzac apparât au milieu d'une liste de philologues: aussi bien Balzac a "étudié» Dante en en proposant une image fictive dans Les Proscrits.

Ce simple constat appelle peut-être à remettre en question la validité de la parole dantesque et surtout de l'idéal herméneutique qu'elle représente: si 
découvrir, en vérité, l'intention de Dante revient à écrire un roman, le livre de Gac est peut-être une critique cruelle de toute entreprise interprétative qui tenterait de reconstruire l'intention de l'auteur.

\section{L'identification à l'auteur: entre roman et folie}

Pour être en apparence éclairant, le discours de Dante sur Dante n'en est pas moins soit le discours d'un fou, soit, ce qui n'est peut-être guère mieux, le discours d'un romancier. On a dit que c'est après un épisode délirant que Dante commence à se confier au docteur Virgilio Pazzi. Or, le traitement proposé par Virgilio à son patient consiste en une transformation de la folie en art romanesque: puisque de nombreux écrivains, notamment des romanciers, se sont pris pour Dante, Dante 2 peut bien faire de même, mais à condition de se présenter comme un romancier, version acceptable de la folie. L'effet métatextuel de cette proposition est foudroyant puisque nous sommes invités à lire le roman de Gac comme le récit d'un fou (Gac) se prenant pour Dante, mais qui a lui-même choisi de se faire passer pour un romancier: le choix de la première personne serait alors moins un choix narratologique qu'un symptôme de schizophrénie et/ou de mégalomanie.

Surtout, le projet herméneutique de s'identifier à un auteur pour mieux comprendre son œuvre devient à son tour pathologico-romanesque. En effet, l'idée de roman ou de folie tire le texte de Gac du discours critique vers le discours expérimental: on ne nous demande guère de prendre au sérieux les révélations de Dante, mais on peut en revanche lire La Guérison comme une tentative de pousser à la limite l'idée d'une critique par identification. Dante réincarné serait alors une figure de tout interprète qui voudrait comprendre Dante en s'identifiant à lui, en étant Dante mais au XX' siècle.

Or, Dante réincarné n'est pas un si bon interprète qu'on pourrait le croire ; ses lectures et ses notations critiques sont bien souvent une caricature de critique biographique. Ainsi, par leur trivialité même, par leur caractère résolument anecdotique, la plupart de ses révélations exagèrent en s'en moquant la curiosité pour l'homme que dissimulerait l'œuvre. Comme le montre in vivo le roman de Gac, l'intimité avec l'auteur n'enrichit pas véritablement le sens de l'œuvre et plus l'on se rapproche des obscures profondeurs de la conscience auctoriale, plus on réduit l'œuvre à une dimension anecdotique. Le projet de l'identification ainsi traité présuppose que l'œuvre reflète une singularité absolue et ne possède aucune dimension universelle. En outre, la forme même choisie par Gac - un récit de vie constellé de citations de 
l'ouvre - n'est pas seulement une imitation de la Vita Nuova, mais aussi une manière de caricaturer la manière de certains biographes et spécialistes de Dante ${ }^{7}$ : ils se contentent d'émailler leur récit de la vie de Dante d'une série de citations tirées de leur contexte qui semblent devoir illustrer le propos biographique. L'œuvre fragmentée et recomposée selon le fil et la logique biographique devient le moyen de dire la vie, au lieu que la vie permette de comprendre l'œuvre. Enfin, la nationalité et le caractère de Dante réincarné, son appartenance au nouveau monde ne font pas seulement révélation, mais sont aussi une manière de souligner, en l'exagérant, l'écart qui sépare l'interprète de la réalité de l'auteur: l'écart qui sépare Dante 2 de Dante $\mathbf{~}$ n'est que le grossissement de la distance qui nous sépare du contexte d'écriture de $L a$ Divine Comédie: face à Dante, nous sommes tous des Chiliens d'origine indienne. C'est encore cette distance que met en valeur l'impuissance de Dante à se comprendre lui-même: "J'ai beau remuer ciel et terre, je ne me souviens pas, mais alors pas du tout, de ce que j'ai voulu dire exactement" (p.9I). Même Dante n'est plus Dante et ne comprend plus Dante. Comment alors prétendre élucider l'œuvre en s'identifiant à l'homme?

De fait, c'est le projet même d'une critique par identification qui se trouve expérimentalement contesté dans le roman de Gac. La tradition herméneutique romantique repose partiellement sur l'idée d'une métamorphose de l'interprète en l'auteur dont le projet sera compris de l'intérieur parce que reproduit, vécu de l'intérieur. C'est par exemple de cette conception que découle cette formule de Sainte-Beuve pour qui compréhension et identification sont une même chose: "C'est toujours avec les expressions dérobées au poète, avec la plume échappée au cygne, que j'écris de lui " (Diaz 1992 : 65). Le même Sainte-Beuve dit explicitement la nécessité d'une fusion du critique en l'auteur: "la critique est pour moi une métamorphose; je tâche de disparaître dans le personnage que je reproduis. Je me fais à lui, même par le style, j'emprunte et je révèle sa diction" (Diaz 43). En somme, pour comprendre l'œuvre, il s'agirait de devenir ici et maintenant l'autre qui vécut ailleurs et jadis. Or, c'est exactement ce projet que met en œuvre le roman de Gac: il décrit ce que serait au XX⿳亠口冋口十 siècle la production, par un être métamorphosé en Dante, d'une Divine comédie. Le résultat est pour le moins

7. Voir par exemple l'introduction de Louis-Paul Guigues à la Vita Nuova, dans l'édition Poésie Gallimard (Guigues 1974). Mais c'est vraisemblablement Boccace (1351) dans sa Vie de Dante, poète florentin qui procède le premier de la sorte, sur le modèle de la vie des auteurs antiques. Cette manière de fragmenter l'œuvre au sein du récit biographique excède évidemment le cas du seul Dante, mais elle est d'autant plus naïve dans le cas de Dante qu'elle reproduit sans distance le dispositif de la Vita Nuova. 
inquiétant: car le résultat de cette métamorphose est un roman, un roman très différent de l'œuvre de Dante, un roman écrit par un auteur qui est Dante mais ne l'est en fait absolument pas. Les exigences d'identification et de reproduction qui président à l'interprétation romantique sont satisfaites mais ne conduisent en rien à une meilleure compréhension du Dante médiéval. Dante 2 ne reproduit pas la démarche de Dante I, alors qu'ils partagent la même identité. Il réécrit, adapte, éclaircit mais ne reconstitue ni la démarche de production ni l'œuvre authentique: «D'ailleurs si j'ai entrepris d'écrire cette nouvelle Comédie, c'est aussi dans le but d'éclaircir et de moderniser la première" (p. 92). Si même Dante ne parvient pas à reproduire sa propre œuvre, qu'en sera-t-il d'un interprète qui a moins de chance d'être une réincarnation de Dante? Or, tout en marquant l'aporie d'un projet interprétatif, Gac propose peut-être conjointement une manière de solution. Car les conseils du docteur Virgilio pourraient concerner, plus que les fous ou les romanciers, les interprètes qui tentent de se métamorphoser en l'auteur qu'ils veulent comprendre. La chose est possible à condition d'accepter son identité soit de fou, ce qui est peu glorieux, soit, ce qui est plus satisfaisant, de romancier: comprendre Dante, se prendre pour Dante reviendrait à écrire un roman où Dante parle à la première personne. Le discours interprétatif par identification est alors possible, mais il est moins un métatexte qu'un hypertexte et moins une reconstruction qu'une invention. Cela n'est pas exactement ce qu'admet Dante réincarné, mais c'est assurément ce qu'a réussi Roberto Gac, interprète de Dante autant que romancier. Donner fictivement la parole à l'auteur sauve l'interprétation d'une folie stérile et fait de la compréhension un art.

Alors le paradoxe touche autant le lecteur que l'auteur: d'abord parce que l'auteur est incapable de se lire, mais surtout parce que l'acte de lecture et de compréhension conduit à se faire auteur d'une fiction, à être soi-même en étant autre, à réinventer non seulement l'œuvre, mais aussi son auteur.

\section{OuVRages CITÉS}

Boccacio, G. (1351) Vie de Dante Alighieri, poète florentin. Traduction de Francisque Reynard. Édition Léo Scheer, 2002.

Borges, J. L. (1949) "El immortal» in El Aleph. Madrid, Alianza Editorial, 1971.

Diaz, J.-L (1992) "Présentation", Sainte-Beuve, Pour la critique. Paris, Gallimard.

Eco, U. (1993) Les Limites de l'interprétation. Paris, Seuil. 
Gac, R. (2000) La Guérison. La Différence.

Guigues, P.L. (1974) «Introduction», Dante, Vita Nuova. Paris, Gallimard, coll. "Poésie».

Lavialle, N. et Puech, J.-B. (eds) (2000) L'Auteur comme ceuvre. L'auteur, ses masques, son personnage, sa légende, Orléans, Presses universitaires d'Orléans, 2000.

Marías, J. (1992) Vidas escritas. Ediciones Siruela, 1992.

Michon, p. (199I) Rimbaud le Fils. Paris, Gallimard, coll. "L'un et L'autre».

Rabau, S. et Dubel, S. (éd.) (200r) Fiction d'auteur? Le discours biographique sur l'auteur de l'Antiquité à nos jours. Paris, Champion.

Schleiermacher (I829) "Discours prononcés à l'Académie" in Herméneutique. Traduit de l'allemand par Christian Berner. Paris, CERF. Schwob, M. (1896) Vies imaginaires. Paris, Éditions Gérard Lebovici, 1986.

Tabucchi, A. (1992) Sogni di sogni. Roma. 